\title{
The Evaluation of Situational Transaction Trust in E-Service Environments
}

\author{
Yan Wang \\ Department of Computing \\ Macquarie University \\ Sydney, NSW 2109 \\ Australia \\ yanwang@ics.mq.edu.au
}

\author{
Ee-Peng Lim \\ School of Information Systems \\ Singapore Management University \\ 80 Stamford Road \\ Singapore 178902 \\ eplim@smu.edu.sg
}

\begin{abstract}
Trust is a critical issue in e-commerce and e-service environments. In some applications (such as eBay), the trust management mechanism has been introduced to provide valuable information to buyers prior to placing orders and making payments. However, most studies and applications focus on determining the general trustworthiness of individuals but not providing transaction specific trust information that involves factors associated with forthcoming transactions. In this paper, we present a new concept - situational transaction trust, and propose a novel approach to evaluate it, which binds existing trust data with a new transaction. This can deliver more accurate trust information to buyers and prevent some typical attacks.
\end{abstract}

\section{Introduction}

In e-commerce or e-service environments, the reputation-based trust status of a seller or a service provider is very important from the point view of a buyer or a service customer. When there are a few sellers or service providers providing the same product or service, the customer would like to order from the seller or service provider with the best transaction reputation. This is particularly important when the customer has to select from unknown sellers or service providers.

In general, in a trust management mechanism enabled system, buyers or service customers can provide feedback and ratings after transactions [13]. The trust management system can calculate the trust value based on collected ratings reflecting the quality of recent transactions. The trust value can be provided to customers, by publishing it on the web or responding to their requests.

Trust issues have been actively studied in Peer-to-Peer (P2P) networks often used for information sharing. In a
P2P system, it is quite natural for a client peer to doubt if a serving peer can provide the complete file prior to any download action, which may be quite time-consuming and network bandwidth-consuming. Different from some trust management system in e-commerce environments, in a P2P trust system, a requesting peer needs to enquire the trust data of a serving peer (target peer) from other peers which may have transacted with the serving peer in the past [1, $5,13]$. The computation of the trust status of the serving peer on the collected trust ratings is then performed by the requesting peer.

In existing trust management studies, the final trust value is computed to reflect the general or global trust status of every service provider. While a buyer or service customer is more concerned about the trust status of transactions of the product or service that the customer is going to order, she or he cannot rely on the global trust, which does not accurately reflect the trust level of the forthcoming transaction. There are some typical attacks that may occur in a trust management enabled environment that provides only global trust status. For example, a malicious seller can abuse the trust management system by selling cheap products and earn good reputation. After having accumulated a good reputation, the seller can start cheating customers by selling expensive products (case 1). In another case (case 2), a malicious seller can cheat customers by offering a much cheaper price to attract mass orders from buyers. After the payment, a buyer may receive nothing.

Thus a good trust management system should provide more (precise) trust information that indicates not only the global trust level, but also the trust status that is bound to the forthcoming transaction. We term this trust as the situational transaction trust (or transaction specific trust [9]), which consists of a set of trust values and extends the notion of situational trust [4] in e-commerce or e-service environments. In this paper, we also propose a novel model for evaluating the situational transaction trust.

This paper is organized as follows. In Section 2, we re- 
view the trust management approaches of eBay and some existing studies. Section 3 discusses the metrics of situational transaction trust evaluation and presents our proposed approach. Some empirical studies are presented in Section 4 for further illustrating the properties of our model. Finally Section 5 concludes our work.

\section{Background}

\subsection{Trust Management at eBay}

eBay.com is a typical Customer-to-Customer (C2C) web site. Its trust management mechanism is one of the earliest systems in applications.

At eBay, after each transaction, the buyer can give feedback to the system according to the service quality of the seller. The feedback (or rating) is stored by eBay (a centralized management architecture). The feedback can be "positive", "neutral" or "negative". eBay calculates the feedback score $S=P-N$, where $P$ is the number of positive feedback left by members (customers) and $N$ is the number of negative feedback from members. $S$ value is displayed on the web page (see Figure 1). $R=\frac{P-N}{P+N}$ (e.g., $R=99.1 \%$ ) is called the positive feedback rate, based on which the seller can be awarded as a "Power Seller" if $R \geq 98 \%(98 \%$ is the threshold).

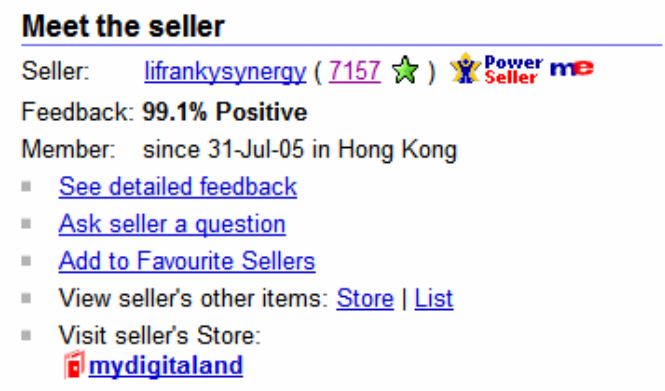

\section{Figure 1. A Seller's Reputation at eBay}

\begin{tabular}{|c|c|c|c|c|c|}
\hline $\begin{array}{l}\text { Feedback Score: } \\
\text { Positive Feedback: }\end{array}$ & $\begin{array}{l}7157 \\
99.1 \%\end{array}$ & \multicolumn{2}{|c|}{ Recent Feedback Ratings } & (last 12 months) & (?) \\
\hline $\begin{array}{l}\text { Members who left a positive: } \\
\text { Members who left a negative: }\end{array}$ & $\begin{array}{l}7225 \\
68\end{array}$ & (4) Positive & $\begin{array}{c}1 \text { month } \\
583\end{array}$ & $\begin{array}{c}6 \text { months } \\
4344\end{array}$ & $\begin{array}{c}12 \text { months } \\
6427\end{array}$ \\
\hline All positive Feedback: & 7928 & () Neutral & 13 & 76 & 103 \\
\hline Find out what these numbers & & $\Theta$ Negative & 4 & 43 & 63 \\
\hline
\end{tabular}

\section{Figure 2. A Seller's Trust Data at eBay}

eBay further provides rating data in 12 months listed in a table, which is divided by recent 1 month, 6 months and 12 months (refer to Figure 2). Thus, eBay provides some simple mechanisms of trust management and trust calculation and leave some raw data to buyers for self-judgement.
It leaves much room for improvement - such as, recent ratings are more important in the trust evaluation [14]. It does not divide sellers into multiple classes of reputation ranks either (e.g. a 5-star seller, or a 4-start seller) as in [6].

\subsection{Other Related Studies}

P2P network technology has been widely used in information-sharing systems, where a peer (serving peer) can share some information (e.g. music files or movie files) with other peers (client peers) without any central management authority. In this environment, the issue that the file being provided is complete or incomplete may be the concern of a client peer before any download action. EigenTrust [1] collects the local trust values of all peers to calculate the global trust value of a given peer. In [5], Marti and Garcia-Molina proposed a voting reputation system targeting at e-commerce applications that collects responses from other peers on a given peer. The final reputation value is calculated by combining the values returned by responding peers and the requesting peer's experience with the given peer.

In the literature, trust issue also received much attention in service-oriented computing research. In [3], Lin et al proposed a method of reputation-based trust evaluation in service-oriented environments based on a proposed architecture consisting of distributed trust management brokers. In [7], Vu et al proposed a model to evaluate and rank the trust and reputation of QoS-based services. In [10], an event-driven and rule-based trust management for serviceoriented application is proposed, where a formula based approach is adopted for incremental trust computation. The approach is adaptable to applications by incorporating rule management. The computed result can be taken as a global trust value reflecting the accumulated trust level, which is not particularly relevant to a forthcoming transaction.

\subsection{Some Problems in Existing Work}

To the best of our knowledge, the existing studies on trust evaluation neglect some important factors that are directly relevant to a buyer. The trust management system should provide precise trust information that can clearly indicate the trust status for a forthcoming transaction based on previous transactions and their trust values. Namely, different situations require different considerations with regard to trust, and hence with different values for trust [4]. With different forthcoming transactions, situations will be different for the same seller or service provider with the same existing trust data set. In this paper, we therefore consider the following:

1. The trust evaluation should offer trust status directly relevant to the product that the buyer is going to purchase. 
In general, in existing studies, the global trust level is computed, which is based on all transactions and corresponding trust values (in a certain period). This is useful but too limited as the trust status of transactions bound to a product is one of the concerns of the buyer. The trust evaluation mechanism should offer clear information. Otherwise, some malicious sellers may abuse the trust management system as we introduced in Section 1. The buyer may have to ignore a seller with good global trust if its transaction trust for the product to be purchased is not good.

Similarly, transactions can also be categorized by products. The trust evaluation should also consider previous transactions in the same product category. This helps offer situational trust to the buyer. For example, when a buyer is going to purchase a digital camera from a seller, the buyer may be concerned about all transactions of the same seller selling various digital cameras, in addition to the transactions of the seller selling the specific digital camera to be ordered. If there are problems in either product level or product category level trust, the buyer may look for other sellers.

2. The trust evaluation should also consider transaction amount (or price). Different transaction amounts may indicate different risk with respect to the loss of a buyer in case of transaction failure. Transaction amounts can be categorized. Transactions in the same amount category can be considered relevant when calculating a transaction trust bound to a new transaction.

Furthermore, the offered price itself may imply the transaction trust level to some extent when compared with the market price, which is considered as reasonable. If the offered price is much higher or less than the market price, it suggests low trust level and high possibility of monetary loss of the buyer or service customer.

In this paper, we propose the new concept - situational transaction trust, and a novel trust evaluation approach. The trust evaluation is based on old transactions and the new transaction that may happen between the buyer and the seller, and incorporates the above discussed factors.

\section{Trust Evaluation}

As mentioned in Section 2.3, from the point view of a buyer, the concern of the trust of a seller should not be the global one only. The trust value should be bound to the particular product or service that the buyer is going to purchase. Namely, it is the situational transaction trust evaluation, which delivers more valuable information to the customer.
In the next subsection, we will present some trust evaluation metrics, based on which we will propose the trust evaluation method.

\subsection{Trust Evaluation Metrics}

\section{Global Trust (GT)}

The global trust value is based on all transactions completed between the service provider or seller with all customers. It helps provide a global overview of the trust status of the service provider or seller. In most existing studies, the final result belongs to global trust type.

In the computation of global trust, recent transactions should be given higher weight. This principle has been followed in many existing studies, such as $[14,11]$.

\section{Product/Service Specific Trust (PST)}

This trust is based on the trust ratings of transactions of the same product or service as this is directly the concern of a buyer or service customer.

\section{Product/Service Category Trust (PCT)}

This trust is is derived based on transactions that involve the same category of the product or service to be ordered.

This trust can illustrate the trust status of the seller or service provider for the quality in the same service (or product) category.

For example, when a buyer is going to buy a digital camera from a seller, the buyer is more likely to trust the seller more if the seller obtained good reputation in all transactions selling various digital cameras.

\section{Transaction Amount Category Specific Trust (TACT)}

The trust of transactions with similar transaction amount (or price) to the new transaction should be computed. It can illustrate the trust status of the seller or service provider for the transactions with the same nature in terms of transaction amount.

\section{Global Weighted Trust (GWT)}

$G W T$ is an important indication for trust level. Its value is bound to all transactions with different transaction amounts $[2,8]$. Namely, when calculating the $G W T$ value, both transaction ratings and transaction amounts are taken into account. The amount (or price) of the new transaction is also taken into account.

This trust value is global in the sense that it is based on all transactions in a recent period. It is termed as the weighted trust as it is bound to the forthcoming 
transaction. The weight depends on the price (transaction amount) of the new transaction, the price of each existing transaction, and the proportion of transactions with the same price as the new transaction in all previous transactions. If most previous transactions of the seller has similar transaction amount as the new one, the $G W T$ value will be good provided that each transaction rating is good.

By calculating the global weighted trust value, the malicious seller mentioned in case 1 in Section 1 can be identified as its trust value will be low, based on the fact that he/she has larger number of transactions with low transaction amount and none or few transactions with the same amount as the new transaction (very expensive one).

\section{Price Trust (PT)}

Furthermore, another concern is also bound with the new transaction - we term it as Price Trust (PT). In general, a buyer is concerned about whether the offered price is a normal one in the market. If it is too high or too low, it indicates high transaction risk level and thus low transaction trust. In particular, some malicious sellers aim to attract buyers by offering quite low price then cheat them. The Price Trust is expected to identify this type of cases (case 2 in Section 1) and leave risk indication to potential buyers.

\subsection{Trust Data Representation}

In order to calculate the situational transaction trust, we assume the following trust data structure.

$$
T R=<S, C, R_{C \rightarrow S}^{(t)}, P, p, t>
$$

where

1. $T R$ is the transaction occurred at time $t$ between seller or service provider $S$ and buyer or service customer $C$;

2. $P=p d(T R)$ is the product purchased in the transaction;

3. $p=\operatorname{pr}(T R)$ is the price for the product or service purchased in the transaction;

4. $R_{C \rightarrow S}^{(t)}=\operatorname{rating}(T R) \in[0,1]$ is the rating given by $C$;

5. In addition, we use $\operatorname{tran}\left(R_{C \rightarrow S}\right)$ to denote transaction $T R$.

\subsection{Global Trust}

As we discussed above, this is not a new issue.

Given the latest period $\left[t_{\text {start }}, t_{\text {end }}\right]\left(t_{\text {end }}+1=\right.$ now $)$, the global trust of seller $S$ is

$$
G T_{S}^{\left[t_{\text {start }}, t_{\text {end }}\right]}=\frac{1}{n} \sum_{t \in\left[t_{\text {start }}, t_{\text {end }}\right]} R_{i}^{(t)} \cdot w_{i}^{(t)}
$$

where

1. $n$ is the number of transactions of $S$ in the period of $\left[t_{\text {start }}, t_{\text {end }}\right]$;

2. $w_{i}^{(t)}$ is the weight for transaction $\operatorname{tran}\left(R_{i}^{(t)}\right)$ at time $t$;

3. $w_{i}^{\left(t_{1}\right)}<w_{j}^{\left(t_{2}\right)}\left(t_{1}<t_{2}\right)$ and $\sum_{t \in\left[t_{\text {start }}, t_{\text {end }}\right]} w_{i}^{(t)}=1$.

\subsection{Product/Service Specific Trust}

In the computation of Product/Service Specific Trust, the transactions selling the same product/service are taken into account.

Definition 1: Let $P$ denote the product/service that customer $C$ is going to order from service provider $S$ at time $t_{\text {end }}+1$. $R_{i}^{(t)}$ denotes the trust rating given to $S$. The Product/Service Specific Trust is defined as follows:

$$
\begin{gathered}
P S T_{S(P)}^{\left[t_{\text {start }}, t_{\text {end }}\right]}= \\
\frac{1}{m} \sum_{p d\left(\operatorname{tran}\left(R_{i}^{(t)}\right)\right)=P \text { and } t \in\left[t_{\text {start }}, t_{\text {end }}\right]} R_{i}^{(t)} \cdot \check{w}_{i}^{(t)}
\end{gathered}
$$

where

1. $m$ is the number of all transactions of $S$ selling $P$ during the period $\left[t_{\text {start }}, t_{\text {end }}\right]$;

2. $\check{w}_{i}^{(t)}$ is the weight for transaction $\operatorname{tran}\left(R_{i}^{(t)}\right), \check{w}_{i}{ }^{\left(t_{1}\right)}<$ $\check{w}_{j}^{\left(t_{2}\right)}\left(t_{1}<t_{2}\right)$, and $\sum_{t \in\left[t_{\text {start }}, t_{\text {end }}\right]} \check{w}_{i}^{(t)}=1$. Note for the same $S$, as $n$ may not be equivalent to $m$, it is likely $w_{i}^{(t)} \neq \breve{w}_{i}^{(t)}$.

\subsection{Product/Service Category Specific Trust}

In the computation of this trust, the transactions selling the products/services in the same category as the new transaction are taken into account. The product/service category can be pre-defined or specified by the customer.

Definition 2: Assume $P$ is the product or service that customer $C$ is going the order from $S$ and $\tilde{C a t}(P)$ denotes the product/service category of $P$. Let $P^{\prime}=\operatorname{pd}\left(\operatorname{tran}\left(R_{i}^{(t)}\right)\right)$ denote the product of transaction $\operatorname{tran}\left(R_{i}^{(t)}\right)$. The Product/Service Category Specific Trust is defined as follows.

$$
P C T_{S(P)}^{\left[t_{\text {start }}, t_{\text {end }}\right]}=\frac{1}{g} \sum_{P^{\prime} \in \tilde{C a}(P)} \sum_{\text {and } t \in\left[t_{\text {start }}, t_{\text {end }}\right]} R_{i}^{(t)} \cdot \tilde{w}_{i}^{(t)}
$$

where 
1. $g$ is the number of all transactions of $S$ selling products in $\tilde{C a t}(P)$ during the period $t \in\left[t_{\text {start }}, t_{\text {end }}\right]$;

2. $\tilde{w}_{i}^{(t)}$ is the weight for transaction $\operatorname{tran}\left(R_{i}\right)$ between customer $C_{i}$ and service provider $S$;

3. $\tilde{w}_{i}^{\left(t_{1}\right)}<\tilde{w}_{j}^{\left(t_{2}\right)}\left(t_{1}<t_{2}\right)$ and $\sum_{t \in\left[t_{\text {start }}, t_{\text {end }}\right]} \tilde{w}_{i}^{(t)}=1$.

\subsection{Transaction Amount Category Specific Trust}

In addition to the product/service category trust, the customer may be concerned about the trust of transaction with similar transaction amount with he new transaction. From the point view of a seller, more transaction amount may imply more profit. However, for the point view of a customer, more transaction amount indicates more loss in case of fraudulent transactions. The trust status of the seller in all transactions with similar transaction amount to the new transaction can outline the reputation and risk level of the seller.

Definition 3: Assume $o p$ is the offered price of the product or service $P$ that customer $C$ is going the order from $S$ and $\hat{C a t}(o p)$ denotes the price category of op. Let $p=\operatorname{pr}\left(\operatorname{tran}\left(R_{i}^{(t)}\right)\right)$ denote the transaction amount (price) of transaction $\operatorname{tran}\left(R_{i}^{(t)}\right)$. The Transaction Amount Category Specific Trust (TACT) is defined as follows.

$$
T A C T_{S(P)}^{\left[t_{\text {start }}, t_{\text {end }}\right]}=\frac{1}{k} \sum_{p \in \hat{\text { Cat }}(\text { op })} \sum_{\text {and } t \in\left[t_{\text {start }}, t_{\text {end }}\right]} R_{i}^{(t)} \cdot \hat{w}_{i}^{(t)}
$$

where

1. $k$ is the number of all transactions of $S$ selling products with price $p \in \hat{C a t}(o p)$ during the period $t \in$ $\left[t_{\text {start }}, t_{\text {end }}\right]$;

2. $\hat{w}_{i}^{(t)}$ is the weight for transaction $\operatorname{tran}\left(R_{i}\right)$;

3. $\hat{w}_{i}^{\left(t_{1}\right)}<\hat{w}_{j}^{\left(t_{2}\right)}\left(t_{1}<t_{2}\right)$ and $\sum_{t \in\left[t_{\text {start }}, t_{\text {end }}\right]} \hat{w}_{i}^{(t)}=1$.

\subsection{Global Weighted Trust}

The aim of the Global Weighted Trust is to illustrate the trust status with respect to the proportion of the same type of transactions as the new transaction among all conducted transactions. The nature of this trust value is depicted as follows.

1. The Global Weighted Trust is high if the number of conducted transactions similar to the new transaction $T R_{n e w}$ is in large proportion of all conducted transactions and obtained trust values are high;
2. The Global Weighted Trust is low if the conducted transactions similar to the new transaction $T R_{n e w}$ are in small proportion among all conducted transactions even if each transaction rating obtained is good.

The calculation of the global weighted trust can be based on the difference of the price of the new transaction (of product $P$ ) and the one of the existing transaction. Namely, $\Delta=o p-$ price $_{\text {old }}$. The different results in the impact factor $\theta \in[0,1]$, which is proportional to the difference. The global weighted trust can be calculated as

$$
G W T_{S(P)}^{\left[t_{\text {start }}, t_{\text {end }}\right]}=\frac{1}{n} \sum_{t \in\left[t_{\text {start }}, t_{\text {end }}\right]} \theta_{i} \cdot R_{i}^{(t)} \cdot w_{i}
$$

where

1. $n$ is the number of all previous transactions with trust ratings;

2. $R_{i}^{(t)}$ is the trust rating for the $i$ th transaction with price $p_{i}$;

3. $\operatorname{Cat}(\cdot)$ is the category of price difference, $\Delta_{i}=o p-p_{i}$ and $\theta_{i}=f\left(\operatorname{Cat}\left(\Delta_{i}\right)\right) \in(0,1]$. When $\Delta_{i}=0, \theta_{i}=1$.

This approach is different from the study in [8] and [12], which categorizes prices and calculates the difference of price categories. The new method is better as in the original method, if $\operatorname{Cat}(\$ 100)=1$ and $\operatorname{Cat}(\$ 101)=2$, then the category difference is $\Delta=$ $2-1=1$. But actually the price difference is only $\$ 1$. In the new method, as $\Delta=1, \operatorname{Cat}(\Delta)=0$.

4. $w_{i}$ is the weight for $R_{i}$ and $\sum_{n} w_{i}=1$.

In an example category, $\$ 100$ difference makes sense to have a new category. E.g. if $\Delta \leq 100, \operatorname{Cat}(\Delta)=1$ and if $100<\Delta \leq 200, \operatorname{Cat}(\Delta)=2$, etc. Also, to represent negative difference, we assume $\operatorname{Cat}(\Delta)=-1$ if $-100 \leq \Delta<0, \operatorname{Cat}(\Delta)=-2$ if $-200 \leq \Delta<-100$, etc. But $\operatorname{Cat}(\Delta)$ is dependent on application domains (e.g. the category in the property market is different from that of electronic appliances). Thus we can assume in a certain domain, there are $D_{1}+D_{2}+1$ price difference categories, i.e. $\operatorname{Cat}(\Delta) \in\left[-D_{1}, D_{2}\right]$

Meanwhile, in order to indicate higher risk of the cheating case mentioned above, if $\left|\Delta_{1}\right|=\left|\Delta_{2}\right|, \Delta_{1}>0$ and $\Delta_{2}<0$, then $\theta_{1}<\theta_{2}$. Thus, if the new transaction price is much higher than existing transaction prices, the calculated $G W T$ is a very low value even if the rating of each finished transaction is very good (e.g. 1.0).

Definition 4: Let $o p$ and price $_{i}$ denote the offered price of the new transaction and the price of existing transaction $i$. 
$\Delta_{i}=o p-$ price $_{i}$ and $\operatorname{Cat}\left(\Delta_{i}\right) \in\left[-D_{1}, D_{2}\right]\left(D_{i}>0\right.$ is an integer). The impact factor is calculated as

$\theta_{i}= \begin{cases}\frac{2}{\frac{10 \cdot \operatorname{Cat}\left(\Delta_{i}\right)}{\alpha \cdot D_{2}}}+e^{-\frac{10 \cdot \operatorname{Cat}\left(\Delta_{i}\right)}{\alpha \cdot D_{2}}} & \text { if } \operatorname{Cat}\left(\Delta_{i}\right) \geq 0 \\ \frac{e^{\frac{10 \cdot \operatorname{Cat}\left(\Delta_{i}\right)}{\alpha \cdot D_{1}}}+e^{-\frac{10 \cdot \operatorname{Cat}\left(\Delta_{i}\right)}{\alpha \cdot D_{1}}}}{e^{\frac{10}{2}}}(1-\beta)+\beta & \text { if } \operatorname{Cat}\left(\Delta_{i}\right)<0\end{cases}$

where $\alpha \geq 1$ and $\beta \in(0,1)$. The setting of $\alpha$ and $\beta$ values will be studied in section 4.1.

\subsection{Price Trust}

Price trust is used to compare the offered price with the market price. Hight price trust value results from low price difference. High price difference leads to low price trust value. This trust value is useful to prevent the fraudulent transaction by offering low and attractive price.

In general, the price trust can be computed from the distance of offered price and the market price. Namely, if the offered price is much lower than the market price, it indicates a low price trust and a high transaction risk. Meanwhile, if the offered price is much higher than the market price, it also certainly indicates a high transaction risk and low price trust. Thus, the offered price should be close to the market price with the distance in a certain range.

Let $\delta_{p}=\frac{o p-m p}{m p}$ denote the price distance in percentage, where $o p$ is the offered price and $m p$ is the market price. Some principles for calculating the price trust are listed as follows.

1. $P T$ is in reverse proportion to $|\delta|$;

2. If $\left|\delta_{p_{1}}\right|=\left|\delta_{p_{2}}\right|, \delta_{p_{1}}<0$ and $\delta_{p_{2}}>0, \delta_{p_{1}}$ should result in a price trust no better than that of $\delta_{p_{2}}$;

This principle aims to identify a case of cheating by offering very low price. For example, normally it is quite difficult to sell a product $50 \%$ lower than the market price. In this case, it is more likely to be a cheating case that the buyer will not receive the product ordered. But it is a bit normal to offer a price $50 \%$ higher than the market price. This is likely for the seller to earn more money instead to cheating the buyer.

Definition 5: Let $o p$ and $m p$ denote the offered price of $S$ and the market price for product $P$ respectively and $\delta_{p}=\frac{o p-m p}{m p}$. The price trust $P T$ is calculated as

$P T_{S(P)}= \begin{cases}\frac{2}{e^{|\delta p| * \gamma}+e^{-\left|\delta_{p}\right| * \gamma}} & \text { if } \delta_{p} \geq 0 \\ \frac{e^{\nu \cdot\left(2 \cdot p_{p}+1\right)}-e^{-\nu \cdot\left(2 \cdot \delta_{p}+1\right)}}{2 \cdot\left(e^{\nu \cdot\left(2 \cdot \delta_{p}+1\right)}+e^{-\nu \cdot\left(2 \cdot \delta_{p}+1\right)}\right)}+0.5 & \text { if } \delta_{p} \in[-1,0)\end{cases}$

where $\gamma \geq 1$ and $\nu \geq 1$ are arguments for controlling the function curve. The setting of $\gamma$ and $\nu$ values will be studied in section 4.2.
According to the above definition, as the function is transformed from the Hyperbolic Secant (in $(0,1])$ and Hyperbolic Tangent (in $(-1,1)$ ) functions respectively, $P T$ is in the scope of $(0,1]$. Other properties will be studied in Section 4.2.

In a centralized architecture (like eBay), as all sellers or service providers are providing services and make transactions on the same web server, it is easy to collect all prices for a certain product or service. In a simple case, the market price of product $P$ can be calculated as the mean of all prices offered to customers. Namely,

$$
m p_{P}=\frac{1}{h} \sum_{S_{i} \text { is a seller of } P} o p_{i}
$$

where $h$ is the number of sellers selling $P$.

With $m p_{P}$, the PT values can be calculated for each price, after which the new market price can be computed after filtering out some less trustworthy prices.

$$
m p_{P}^{\prime}=\frac{1}{h^{\prime}} \sum_{P T\left(o p_{i}\right) \geq \rho} o p_{i}
$$

where $h^{\prime}$ is the number of sellers whose price trust is no worse than a threshold $\rho$, i.e. $P T\left(o p_{i}\right) \geq \rho$.

The calculation of price trust is an iterative process as with the new market price, the PT value for each offered price will be re-calculated. This iterative process can be repeated until each value becomes stable.

\subsection{Situational Transaction Trust}

Based on the above discussion, the situational transaction trust can be represented in a vector as follows.

Definition 6: Given a seller or service provider $S$ selling a product or service $P$ with price $p$, its situational transaction trust can be defined as a 6-tuple:

$$
\begin{gathered}
S T T_{S(P)}^{\left[t_{\text {start }}, t_{\text {end }}\right]}= \\
<G T_{S(P)}^{\left[t_{\text {start }}, t_{\text {end }}\right]}, P S T_{S(P)}^{\left[t_{\text {start }}, t_{\text {end }}\right]}, P C T_{S(P)}^{\left[t_{\text {start }}, t_{\text {end }}\right]}, \\
T A C T_{S(P)}^{\left[t_{\text {start }}, t_{\text {end }}\right]}, \operatorname{GWT}_{S(P)}^{\left[t_{\text {start }}, t_{\text {end }}\right]}, P T_{S(P)}^{\left[t_{\text {start }}, t_{\text {end }}\right]}>
\end{gathered}
$$

Now the concern is how to compare the trust of two sellers or service providers. In this section, we define two comparison methods: strong comparison and weak comparison. Definition 7: Given two sellers or service providers $A$ and $B$ selling the same product/service $P, A$ is strongly better than $B$ if and only if for each element $T$ in $S T T_{S(P)}, T_{A}>$ $T_{B}$. It is denoted as $S T T_{A}>_{s} S T T_{B}$.

Definition 8: Given two sellers or service providers $A$ and $B$ selling the same product/service $P, A$ is weakly better than $B$ if and only if $P S T_{A}>P S T_{B}$, and $P T_{A}>P T_{B}$. It is denoted as $S T T_{A}>_{w} S T T_{B}$.

Similarly, the relational operators $\geq_{s}$ and $\geq_{w}$ can be defined. Due to the space constraint, we ignore the definitions. 


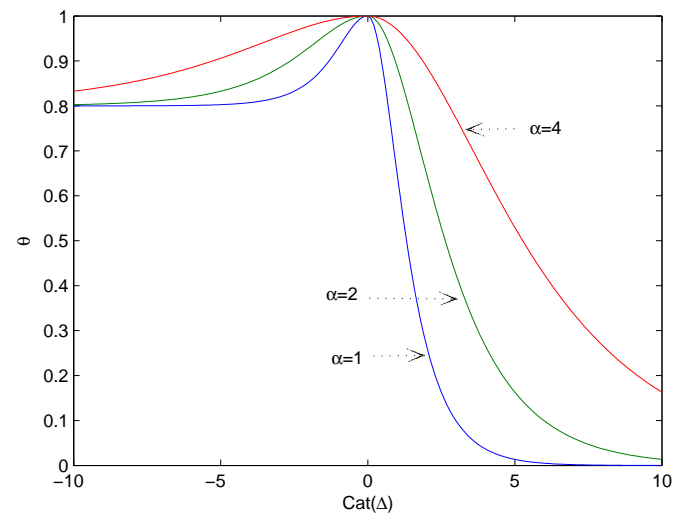

Figure 3. $\theta(\beta=0.8)$

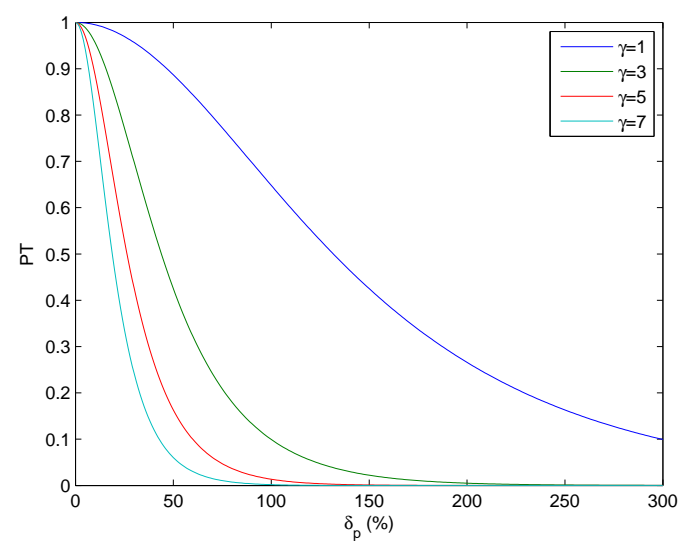

Figure 4. PT when $\delta_{p} \geq 0$

\section{Empirical Study}

Formulae (7) and (8) are defined with arguments. The selection of arguments will be studied in this section.

\subsection{Study 1}

In this section, we study the properties of formula (7) to calculate the GWT. We assume $D_{i}=10$, namely, $\operatorname{Cat}(\Delta) \in[-10,10]$. We set $\alpha=1,2,4$ respectively and $\beta=0.8$.

The result in Figure 3 illustrates that a smaller $\alpha$ value leads to a sticker curve. When $\Delta<0$ and $\operatorname{Cat}(\Delta)=-D_{1}$ $\left(D_{i}=10\right), \theta$ is close to $\beta$.

\subsection{Study 2}

In this section, we study the properties of formula (8) to calculate the Price Trust. We set $\gamma=1,3,5$, or $7, \nu=1,2$, or 3 and observe the function curve changes.

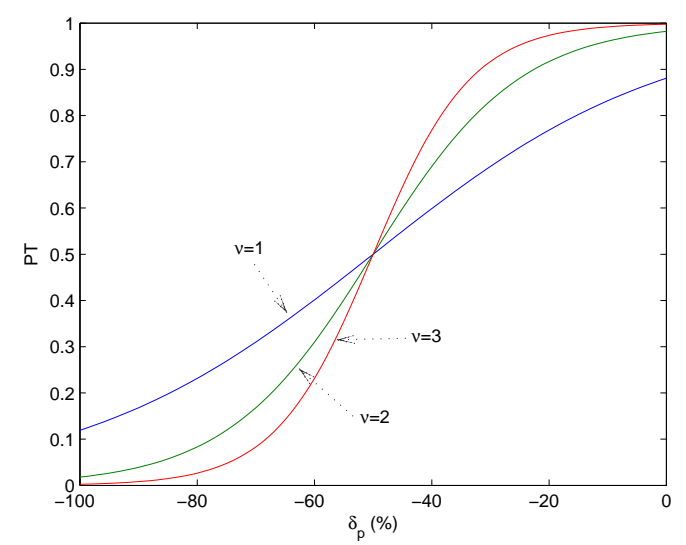

Figure 5. PT when $\delta_{p} \leq 0$

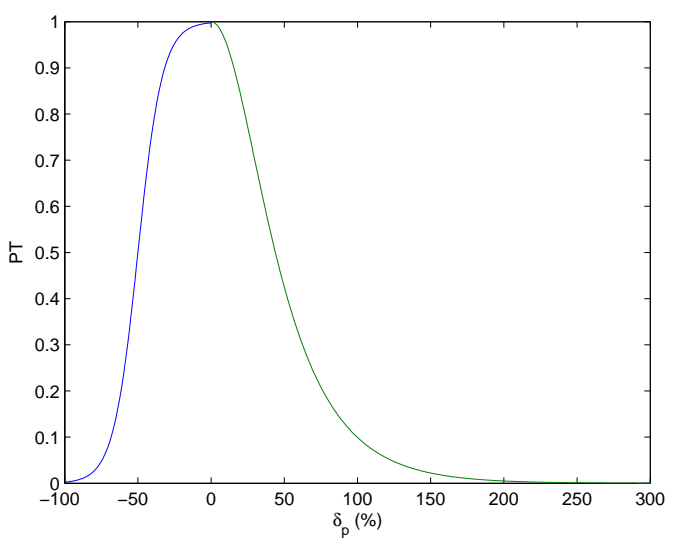

Figure 6. PT when $\delta_{p} \leq 0(\gamma=3, \nu=3)$

In Figure 4 , when $\delta_{p}=50 \%, P T=0.89,0.44,0.17$, or 0.064 if $\gamma=1,3,5$, or 7 respectively. When $\delta_{p}=100 \%$, $P T=0.65,0.1,0.014$, or 0.002 if $\gamma=1,3,5$, or 7 respectively. Relatively, the result is more reasonable when $\gamma \in[3,5]$.

Similarly, the function curve changes can be observed in Figure 5 for the case of $\delta_{p} \leq 0$ when setting $\nu=1,2$ or 3 . A larger $\nu$ value results in sleeper curve. We can observe that when $\nu=2$ or 3 , the trust value is close to 1.0 if $\delta_{p}=10 \%$ or $20 \%$. But PT starts to drop when $\delta_{p}$ is more than $30 \%$. This is reasonable to reflect the nature of price trust.

When selecting $\gamma=3$ and $\nu=3$, formula (8) is plotted in Figure 6.

\subsection{Study 3 - Two Examples}

In this section we introduce a case study with two examples.

In Table 1 , the $S T T$ values of three service providers providing product $P_{1}$ are listed. It is easy to observe that 
$S T T_{S_{1}} \geq_{s} S T T_{S_{2}} \geq_{s} S T T_{S_{3}}$. Therefore, $S_{1}$ is the best one to have the transaction.

But as there are six values in the $S S T$ tuple, sometimes the partial order, not the total order, exists in the service provider set.

\begin{tabular}{ccccccc}
\hline Service Providers & $G T$ & $P S T$ & $P C T$ & $T A C T$ & $G W T$ & $P T$ \\
\hline$S_{1}$ & 0.95 & 0.98 & 0.98 & 0.98 & 0.95 & 0.98 \\
$S_{2}$ & 0.95 & 0.98 & 0.98 & 0.98 & 0.85 & 0.95 \\
$S_{3}$ & 0.85 & 0.88 & 0.86 & 0.82 & 0.85 & 0.90
\end{tabular}

Table 1. STT values of three service providers

In Table 2, we can observe that $S T T_{S_{4}}>_{w} S T T_{S_{6}}$, $S T T_{S_{5}}>_{w} S T T_{S_{6}}, S T T_{S_{6}}>_{w} S T T_{S_{7}}$ and $S T T_{S_{6}}>_{w}$ $S T T_{S_{8}}$. But it is not a full order set. In this case, the judgement to select the best seller has to be left to the customer according to the customer's preference. For example, each customer can specify a set of weights $w_{i}$ where $w_{i}$ is the weight for the $i t h$ element $T_{i}$ in the trust vector. All service providers can be ordered by the final trust value $T=\frac{1}{n} \Sigma_{i} T_{i} \cdot w_{i}$, from which the best service provider can be selected.

\begin{tabular}{ccccccc}
\hline Service Providers & $G T$ & $P S T$ & $P C T$ & $T A C T$ & $G W T$ & $P T$ \\
\hline$S_{4}$ & 0.93 & 0.94 & 0.98 & 0.98 & 0.95 & 0.98 \\
$S_{5}$ & 0.92 & 0.96 & 0.98 & 0.98 & 0.85 & 0.95 \\
$S_{6}$ & 0.95 & 0.88 & 0.86 & 0.82 & 0.85 & 0.90 \\
$S_{7}$ & 0.85 & 0.86 & 0.86 & 0.82 & 0.85 & 0.85 \\
$S_{8}$ & 0.85 & 0.84 & 0.86 & 0.82 & 0.85 & 0.87 \\
\hline
\end{tabular}

Table 2. $S T T$ values of five service providers

\section{Conclusions}

In this paper, we present the new concept of situational transaction trust and an approach for trust evaluation. The trust result is a vector consisting of six trust values, which can outline not only the global trust level, but also the specific trust level that is particularly bound to a forthcoming transaction. This result is especially important and valuable to a buyer or a service provider prior to the transaction. To the best of our knowledge, this is the first work to address the situational transaction trust in detail.

In the future work, more factors can be incorporated for the trust evaluation, such as the reputation of the web site. This may include the policy for transaction management and disputation solving, and security technology for transactions. In addition, if the customer already has some experience with some sellers, the proposed approach should be incorporated with the experience for trust evaluation and decision making.

\section{References}

[1] S. D. Kamvar, M. T. Schlosser, and H. Garcia-Molina. The eigentrust algorithm for reputation management in $\mathrm{P} 2 \mathrm{P}$ networks. In Proceedings of the 12th International WWW Conference, Budapest, Hungary, May 2003.

[2] K.-J. Lin, J. Y. Hsu, Y. Zhang, and T. Yu. A distributed reputation broker framework for web service applications. Journal of E-Commerce Research, 7(3), 2006.

[3] K.-J. Lin, H. Lu, T. Yu, and C. en Tai. A reputation and trust management broker framework for web applications. In Proceedings of The 2005 IEEE International Conference on e-Technology, e-Commerce and e-Service (EEE'05), pages 262-269, March 2005.

[4] S. Marsh. Formalising Trust as a Computational Concept. University of Stirling, 1994.

[5] S. Marti and H. Garcia-Molina. Limited reputation sharing in P2P systems. In Proceedings of ACM EC'04, pages 91101, New York, USA, May 2004.

[6] S. Song, K. Hwang, R. Zhou, and Y.-K. Kwok. Trusted $\mathrm{p} 2 \mathrm{p}$ transactions with fuzzy reputation aggregation. IEEE Internet Computing, 9(6):24-34, 2005.

[7] L.-H. Vu, M. Hauswirth, and K. Aberer. Qos-based service selection and ranking with trust and reputation management. In Proceedings of 13th International Conference on Cooperative Information Systems (CoopIS 2005), Oct 31-Nov 4 2005.

[8] Y. Wang and F. Lin. Trust and risk evaluation of transactions with different amounts in peer-to-peer e-commerce environments. In Proceedings of The IEEE International Conference on e-Business Engineering (ICEBE 2006), pages 102109, Shanghai, China, October 2006.

[9] Y. Wang and K.-J. Lin. Reputation-oriented trustworthy computing in e-commerce environments. IEEE Internet Computing, (July/August):55-59, 2008.

[10] Y. Wang, K.-J. Lin, D. S. Wong, and V. Varadharajan. The design of a rule-based and event-driven trust management framework. In The IEEE International Conference on eBusiness Engineering (ICEBE 2007), pages 97-104, Hong Kong, October 2007.

[11] Y. Wang and V. Varadharajan. A time-based peer trust evaluation in P2P e-commerce environments. In Proceedings of 5th International Conference on Web Information Systems Engineering (WISE'04), volume LNCS 3306, SpringerVerlag, pages 730-735, Brisbane, Australia, November 2004.

[12] Y. Wang, D. S. Wong, K.-J. Lin, and V. Varadharajan. Evaluating transaction trust and risk levels in peer-to-peer ecommerce environments. Journal of Information Systems and E-Business Management (ISeB), 6(1):25-48, 2008.

[13] L. Xiong and L. Liu. PeerTrust: Supporting reputationbased trust for peer-to-peer electronic communities. IEEE Trans. on Knowlege and Data Engineering, 16(7):843-857, 2004.

[14] G. Zacharia and P. Maes. Trust management through reputation mechanisms. Applied Artificial Intelligence Journal, 9:881-908, 2000. 